\title{
Serum IgE Predicts Difference of Population and Allergens in Allergic Diseases: Data from Weifang City, China
}

\author{
Zhang Xu-De $\mathbb{D}^{1},{ }^{1}$ Guo Bei-Bei $\mathbb{D}^{1},{ }^{1}$ Wang Xi-Juan $\mathbb{D}^{1},{ }^{1}$ Li Hai-Bo $\mathbb{D}^{2},{ }^{2}$ Zhang Li-Li $\mathbb{D}^{2}$ \\ and Liu Feng-Xia ${ }^{1}$ \\ ${ }^{1}$ Department of Allergy, The First Affiliated Hospital of Weifang Medical University/Weifang People's Hospital, Weifang, China \\ ${ }^{2}$ Department of Central Laboratory, The First Affiliated Hospital of Weifang Medical University/Weifang People's Hospital, \\ Weifang, China
}

Correspondence should be addressed to Liu Feng-Xia; wf_lfx@163.com

Received 31 December 2020; Revised 8 April 2021; Accepted 9 June 2021; Published 28 June 2021

Academic Editor: Ulrich Eisel

Copyright ( $) 2021$ Zhang Xu-De et al. This is an open access article distributed under the Creative Commons Attribution License, which permits unrestricted use, distribution, and reproduction in any medium, provided the original work is properly cited.

\begin{abstract}
Background. Immunoglobulin E (IgE) is the most important promoter of allergic inflammation. However, there are few systematic studies on IgE in age range, genders, disease spectrum, and time regularity. Aim. To screen the common allergens, allergen spectrum, and IgE difference between type 2 inflammatory allergic diseases and other allergic diseases in Weifang, China. Methods. A retrospective study was performed by estimating patients' clinical data suffering from allergic diseases (urticaria, pollinosis, allergic rhinitis, atopic dermatitis, and bronchial asthma) between May 2019 and April 2020 using an allergen detection kit of Macro-Union Pharmaceutical. Results. 732 of the 1367 patients showed different antigen positive, and the positive rate was $53.5 \%$. The most common allergens were dust mites, mixed fungi, Artemisia pollen, cat/dog dander, and cockroaches. There were $27.0 \%(369 / 1367)$ of the patients with single positive allergen-specific $\operatorname{IgE}(\mathrm{sIgE}), 26.5 \%$ (363/1367) with multiple-positive IgE. The total immunoglobulin E (tIgE) levels varied with gender, age, and type of disease. There was a difference in the distribution of allergens between children and adults. A positive correlation between the serum-specific IgE and the corresponding local inhaled allergen density was observed. Conclusions. In this study, we found that type 2 inflammatory allergic diseases have higher serum IgE and a higher probability of inhaled sIgE positive. According to age, gender, and condition, serological IgE detection of allergens provides new insight into the early diagnosis and prevention of allergic diseases.
\end{abstract}

\section{Introduction}

IgE-mediated allergic diseases are usually multisymptomatic, including allergic rhinitis (AR), allergic asthma (AS), urticaria, atopic dermatitis (AD), and eczema, which have become significant public health issues. The pathogenesis remains largely unknown. Allergic inflammation induced by certain inhaled substances or food antigens in the environment has been implicated in IgE-mediated allergic diseases. Based on the biological mechanisms that underline these diseases, AR, atopic dermatitis, and AS are widely regarded as classic type 2 inflammatory (Th2-dominated response) with the increase of circulating IgE leveleosinophilic inflammation in the human body [1-4]. Long sustained exposure to airborne allergens is known to result in persistent inflammation in AR and AS. Regarding urticaria, IgE-mediated mast cell activation, degranulation, and release of histamine and inflammatory mediators play critical roles in the pathogenesis of allergic diseases [5]. Many studies have suggested that IgE plays a crucial role in immune and inflammatory responses, which is a Th2 biomarker and participates in regulating Th2 inflammatory response.

The prevalence of allergic diseases has been raised due to increased environmental and industrial exposures in recent decades [6-8]. Environmental factors play an important role in the pathogenesis of AR and other respiratory and skin allergic diseases [9-11]. The prevalence of IgE-mediated allergic diseases increased progressively in the developed 
countries, which currently account for $10 \%$ of children subject to food allergy [12], and $40 \%$ of the population with allergic rhinitis $[13,14]$. There are over 330 million asthma patients worldwide [15], which accounts for $20 \%$ of children and $2-18 \%$ of adults among the AD population [16]. However, up to now, the high prevalence of allergic diseases in the population has not been effectively curbed, and people are still plagued by diseases, which indicates that the human understanding of the diagnosis and treatment of such diseases is still insufficient [17-19]. The increased global prevalence of allergic diseases is mainly attributed to environmental factors, suggesting that controlling environmental exposures may protect against allergic diseases in predisposed individuals. Therefore, it is of great significance to identify allergens for the prevention and treatment of allergic diseases. With industrialization development in China, allergic diseases have become a public health concern with increasing incidence. The prevalence of allergic disorders is closely related to various environmental allergens implicated in AR and asthma, including dust mite, mold, pollen, and animal fur $[20,21]$. Although the prevalence and possible causes of AR/AS have been well documented in many developed countries, little information is available in China [22]. Because of the vast territory, different topographic, climatic, and economic conditions, and plant species, allergen spectrum is different from region to region in China. In view of this, we collect data of 1367 patients with allergic diseases, including $\mathrm{AS}, \mathrm{AR}, \mathrm{AD} / \mathrm{eczema}$, and urticaria. In this study, we aim to explore the allergen spectrum of Weifang city in China and investigate the association between allergic reaction and specific allergens, which will thus provide a rationale for the selecting allergens to be tested based on the clinical presentations.

\section{Methods}

2.1. Study Subjects. This retrospective study was approved by the Ethics Review Board of Weifang People's Hospital, Weifang, China. A total of 79 nonatopic subjects and 1367 patients with AR, AS, AD, and urticaria, who received treatment at the Department of Allergy, Respiration and Pediatrics in Weifang People's Hospital between May 2019 and April 2020, were enrolled in the study. Only the first test report was included if the same person met the diagnostic allergen detection multiple times within the same time range. Patients with ambiguous and suspicious diagnoses were excluded. There were 638 males and 729 females aged from 2 months to 87 years old. There were 266 infants aged 0-4 years, 341 school-age children (5-11y) and adolescents $(12-17 y)$, and 760 adults aged over 18 years. Four hundred four cases of urticaria, 345 cases of $\mathrm{AD}$ and eczema, 324 cases of AS and cough variant asthma (CVA), and 233 patients of $\mathrm{AR}$ (including pollinosis) were all included (Table 1).

2.2. Allergen-Specific IgE Antibody Detection. 2-4 ml fresh peripheral venous blood was collected and clotted at room temperature for 20 minutes, centrifuged at $3600 \mathrm{rpm}$ for 4 minutes. The serum was separated and stored at $4^{\circ} \mathrm{C}$ for examination. The inhaled allergens and ingested allergens
TABle 1: Number and percentage of allergic diseases among 1367 patients.

\begin{tabular}{lc}
\hline Diseases & Number (proportions) \\
\hline Urticaria (acute/chronic) & $404(29.6 \%)$ \\
Allergic dermatitis/eczema & $345(25.2 \%)$ \\
Allergic asthma/CVA/chest tightness & $324(23.7 \%)$ \\
Allergic rhinitis/pollinosis & $233(17.0 \%)$ \\
Anaphylactic reaction without clear & $16(1.2 \%)$ \\
predisposing factors & $30(2.2 \%)$ \\
Papular urticaria & $7(0.5 \%)$ \\
Allergic conjunctivitis & $22(1.6 \%)$ \\
Others &
\end{tabular}

were tested using an allergen sIgE antibody detection kit (Beijing Macro-Union Pharmaceutical Co. Ltd., China). The allergen-specific IgE antibody detection kit was used to detect serum sIgE by chemiluminescent immunoassay, whose clinical usefulness was documented compared to other assays for specific IgE measurement [23]. A standard curve was established for quality control. Briefly, 96-well plates coated with allergen were put into the CLIA200 automatic operation platform. $50 \mu \mathrm{l}$ of the sample diluent was added into each well. $50 \mu \mathrm{l}$ of the sample was added to the mix. The coated plate was sealed with the sealing film and incubated in a $37^{\circ} \mathrm{C}$ constant temperature incubator for 45 minutes. After incubation, the vessel was diluted and released. The working solution shall not be less than $350 \mu \mathrm{l}$ for washing, which shall be left for $30 \mathrm{~s}$ and pat dry for five times in total. After washing the plate, we added $100 \mu \mathrm{l}$ enzyme conjugates to each well and passed the plate after incubation. After that, $50 \mu \mathrm{l}$ luminescent substrate $\mathrm{A}$ and substrate B were added into each well, respectively. The vessel was shaken at room temperature for 5 minutes, avoiding light. The luminescent intensity (RLU) of each pore was measured by chemiluminescent immunoassay (Yantai Addcare Bio-Tech Co. Ltd., China). The double logarithm fitting software was used for analysis.

2.3. Diagnosis of Allergic Diseases. In this study, we have defined different allergic diseases simply and effectively according to the international consensus of diagnostics so that researchers can screen them from thousands of medical records: AR: rhinorrhea, nasal obstruction, nasal itching, and sneezing with or without ocular symptoms [24]; AS: recurrent episodes of wheeze, cough, breathlessness, and chest tightness, which are usually associated with variable airflow obstruction and bronchial (airway) hyperresponsiveness [25]; AD: xerosis, pruritus, and erythematous lesions with increased transepidermal water loss [26]; urticaria: the sudden appearance of wheals and/or angioedema, those with a course of more than six weeks are defined as chronic urticaria (CU), or acute urticaria $[27,28]$; eczema: clusters of punctate erythema and needle to miliary-sized papules and papules and herpes, dense patches, basal flush, mild swelling, diffuse boundary, dry skin, and severe itching [29]; papular urticaria: typical local or systemic red papules with constant itching; anaphylactic reaction: the severe allergic reaction that is rapid 
in onset and may cause death including difficulty in breathing and chest tightness, laryngeal edema, and anaphylactic shock [30].

2.4. Statistical Analysis. SPSS version 19.0 software (SPSS Inc., Chicago, IL, USA) was used for data analysis. Positive rate (\%) is the percentage of the positive serum test to various allergens. Different diseases are considered as categorical variables. The chi-square test evaluates the difference among variables. A two-tailed $p<0.05$ is considered to be statistically significant.

\section{Results}

3.1. Age, Gender, and Serum tIgE in All Participants. 1367 patients and 79 nonatopic subjects were included in the study. The patients' mean age was 25.1 years (95\% CI, 24.0 to 26.1 years). There were 267,338 , and 762 patients in the 0-4 (infancy), 5-17y, and adult groups. The tIgE of 934 patients $(68.3 \%)$ exceeded $100 \mathrm{IU} / \mathrm{ml}$. The mean value of $\mathrm{tgE}$ was $225.4 \mathrm{IU} / \mathrm{ml}$ in males and $192.3 \mathrm{IU} / \mathrm{ml}$ in females. There was a significant difference in $\operatorname{tgE}$ between groups regarding gender $(p<0.001)$ (Table 2$)$.

The mean of tIgE in the $0-4$ age group was $190.6 \mathrm{IU} / \mathrm{ml}$ (95\% CI, 173.9 to $207.4 \mathrm{IU} / \mathrm{m})$. The mean value of $\operatorname{tgE}$ in the 5-17 groups was $252.0 \mathrm{IU} / \mathrm{ml}$ (95\% CI, 229.7 to 274.4 $\mathrm{IU} / \mathrm{ml}$ ). The mean value of $\operatorname{tgE}$ in the adult groups was $194.1 \mathrm{IU} / \mathrm{ml}$ (95\% CI, 182.4 to $205.8 \mathrm{IU} / \mathrm{m})$ ). The difference of tIgE was significant between the adolescent and adults $(p<0.001)$. Similar results were found between the infant group and the adolescent group $(p<0.001)$. There were 544 cases of hypersensitivity of the respiratory system. The average total IgE was $263.3 \mathrm{IU} / \mathrm{ml}$. The tIgE of 828 patients only with cutaneous symptoms was $171.7 \mathrm{IU} / \mathrm{ml}$. When we further refined the disease into $\mathrm{AR}, \mathrm{AS} /$ cough/chest distress, acute/chronic urticaria, and eczema/AD (complications excluded), the average $\operatorname{tgE}$ was decreased in turn, and the differences between groups were statistically significant (Figure 1).

3.2. Average Age Associated with Single Positive Allergen in Allergic Disease Patients. As shown in Table 3, the number of allergens of allergic disease patients was as follows: dust mite (394), mixed fungi (266), Artemisia pollen (173), Humulus scandens pollen (123), cockroach (109), dog dander (104), cat dander (89), ragweed pollen (77), milk (49), egg white (42), soybeans (40), tree pollen (poplar/willow/elm) (36), sea fish/crab (30), peanuts (23), and sea shrimp (19). As a result, it could be quickly concluded that dust mites, mixed fungi, Artemisia pollen, and Humulus pollen were the main allergens causing allergic diseases in Weifang, China.

The age of the allergic population varied with different allergens: egg: 7.5 years (95\% CI, 4.3 to 10.7 years), milk: 7.7 years (95\% CI, 4.7 to 10.7 years), peanut: 15.8 years (95\% CI, 9.2 to 22.5 years), mixed fungi: 19.1 years $(95 \%$ CI, 16.9 to 21.3 years), dust mite: 22.4 years (95\% CI, 20.6 to 24.3 years), Artemisia pollen: 24.2 years (95\% CI, 21.4 to 27.0 years), cat: 25.4 years (95\% CI, 21.3 to 29.5 years), dog: 25.5 years (95\% CI, 21.8 to 29.2 years), Humulus scandens: 27.7 years ( $95 \%$ CI, 24.4 to 30.9 years), tree pollen: 30.1 years
(95\% CI, 24.3 to 35.9 years), soybean: 27.4 years (95\% CI, 20.9 to 33.9 years), ragweed pollen: 27.5 years (95\% CI, 23.1 to 31.9 years), and cockroach: 32.4 years (95\% CI, 28.6 to 36.2 years) (Figure 2 ).

3.3. sIgE Level of Inhaled Allergens and Ingestible Allergens in Different Allergic Disease Patients. The level of inhaled sIgE was significantly different between the respiratory allergic disease and the allergic skin disease $(p<0.001)$. In contrast, no statistical differences of sIgE were found between groups of AR and AS, urticaria, and eczema. There was no significant difference of $\operatorname{sigE}$ between the two groups of inhaled allergens and ingestible allergens. Moreover, patients with respiratory allergies had higher levels of sIgE than those with allergic dermatosis, and most patients with respiratory allergies were aeroallergens positive (Figures 3 and 4). There was no significant correlation between $\operatorname{tgE}$ and $\operatorname{sgE}$ in all allergic groups.

3.4. Time Regularity of Allergic Disease Positive with Specific Allergens. The median concentration of sIgE in patients positive with 15 specific allergens in different months of the year was analyzed. There was a peak in March, June, and November for dust mite and mixed fungi, respectively. Also, patients' sIgE level positive with dust mite peaked in August, while Artemisia and Humulus pollen had only one peak in September. The peak of poplar/willow/elm's sIgE appeared in June, while the annual dispersal of tree pollen occurred in May. Accordingly, as reported in the previous study, there was one month time postponement between the air concentration of tree pollen and the corresponding serum sIgE concentration of patients [31]. A positive correlation was found between annual total pollen quantity and median sIgE values (Figure 5). In contrast, the incidence of ingestible allergens, especially regarding peanuts, soybeans, and milk, was most common in March and July (Figure 6).

\section{Discussion}

More and more epidemiological studies have confirmed the objective existence of "atopic march"; consequently, the early prevention and prediction of allergic diseases have become a hot issue [32-35]. Numerous studies have confirmed that antigens can sensitize the body through the damaged epidermal layer, thereby initiating local and systemic immune responses, increasing the incidence of eczema, AR, and AS [36]. It has been proposed that the observed temporal relationship between atopic diseases may help earlier diagnosis and may facilitate novel approaches to prevent the disease $[34,37]$. This study found that the primary manifestation of allergic infants and young children is AD caused by ingestible allergens. Allergic adolescents mainly have respiratory disorders. The sIgE of dust mites and various airborne pollen is gradually detected, which can change with age. Recently, Belgrave et al. concluded that the developmental profiles of eczema, wheeze, and rhinitis were heterogeneous, and only a tiny proportion of children (7\% of those with symptoms) followed trajectory profiles resembling the atopic march [38]. Nevertheless, each region has its unique allergen 
TABLE 2: Characteristics of all participants regarding age and tgE.

\begin{tabular}{|c|c|c|c|c|c|c|}
\hline & \multicolumn{3}{|c|}{ Nonatopic subjects } & \multicolumn{3}{|c|}{ Patients } \\
\hline & Male & Female & Total & Male & Female & Total \\
\hline Patients, no. (\%) & $37(46.8)$ & $42(53.2)$ & $79(100)$ & $638(46.7)$ & $729(53.3)$ & $1367(100)$ \\
\hline $\begin{array}{l}\text { Age range (year } \\
\text { (minimum-maximum)) }\end{array}$ & $7-75$ & $4-76$ & $4-76$ & $0.3-86$ & $0.4-87$ & $0.3-87$ \\
\hline Mean age (y (95\% CI)) & $41.7(36.0-47.5)$ & $36.6(30.6-42.5)$ & $38.2(34.0-42.5)$ & $20.0(18.5-21.6)$ & $29.2(27.8-30.6)$ & $25.1(24.0-26.1)$ \\
\hline $\begin{array}{l}\text { Mean total IgE } \\
(\mathrm{IU} / \mathrm{ml}(95 \% \mathrm{CI}))\end{array}$ & $64.6(45.7-83.5)$ & $63.4(51.5-75.3)$ & $64.0(53.3-74.6)$ & $225.4(211.4-239.5)$ & $192.3(182.2-204.4)$ & $207.8(198.5-217.0)$ \\
\hline $\begin{array}{l}\text { Sensitization to } \\
\text { respiratory allergens, } \\
\text { no. (\%) }\end{array}$ & $0(0)$ & $2(4.8)$ & $2(2.5)$ & $341(53.4)$ & $323(44.3)^{\mathrm{a}}$ & $664(48.6)$ \\
\hline $\begin{array}{l}\text { Sensitization to } \\
\text { ingestible allergens }\end{array}$ & $0(0)$ & $0(0)$ & $0(0)$ & $89(13.9)$ & $63(8.6)^{b}$ & $152(11.1)$ \\
\hline
\end{tabular}

Chi-square test: ${ }^{\mathrm{a}} p=0.001 ;{ }^{\mathrm{b}} p=0.002$.

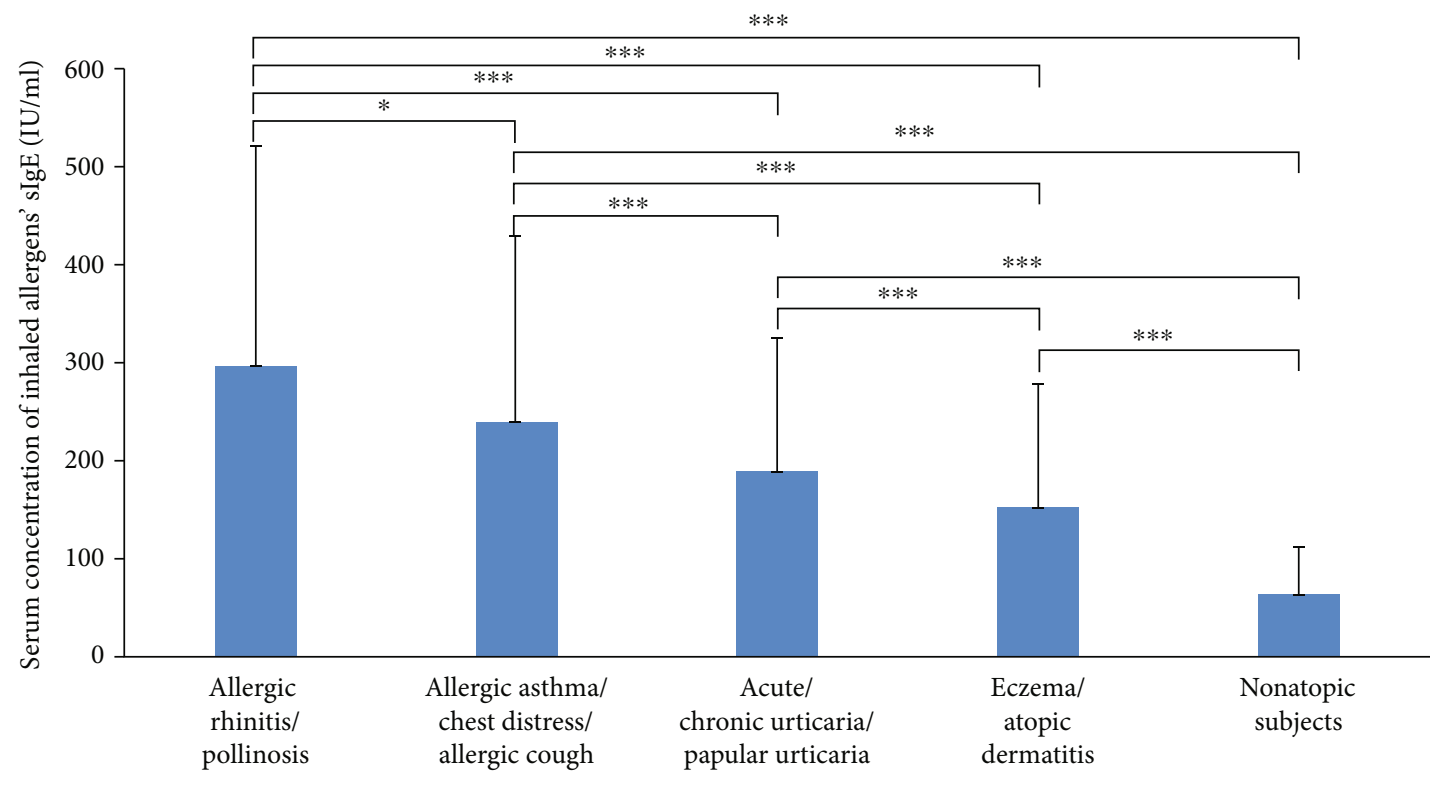

Figure 1: Mean value of total IgE in different diseases $\left({ }^{* *} p=0.024,{ }^{* * *} p<0.001\right)$.

TABLE 3: Number of allergen-positive patients with the relevant allergic diseases.

\begin{tabular}{|c|c|c|c|c|c|c|c|c|c|c|c|c|c|c|c|}
\hline & $\mathrm{Ar}$ & Mite & Dog & Cat & $\mathrm{Cr}$ & $\mathrm{Ra}$ & $\mathrm{Hu}$ & Fungi & Tree & Egg & Milk & Peanut & Soy & Shrimp & Fish \\
\hline *Allergic rhinitis/pollinosis & 48 & 117 & 22 & 19 & 13 & 22 & 33 & 58 & 6 & 0 & 5 & 1 & 7 & 1 & 1 \\
\hline *Allergic asthma/allergic cough/chest tightness & 61 & 130 & 30 & 23 & 28 & 22 & 33 & 95 & 10 & 8 & 7 & 6 & 5 & 1 & 3 \\
\hline${ }^{*}$ Acute/chronic urticaria, papular urticaria & 26 & 83 & 25 & 23 & 33 & 20 & 26 & 54 & 14 & 15 & 19 & 11 & 14 & 6 & 9 \\
\hline${ }^{*}$ Eczema/allergic dermatitis & 30 & 60 & 26 & 24 & 33 & 10 & 25 & 59 & 5 & 19 & 18 & 4 & 12 & 11 & 16 \\
\hline Anaphylactic & 8 & 4 & 1 & 0 & 2 & 3 & 6 & 0 & 1 & 0 & 0 & 1 & 2 & 0 & 1 \\
\hline
\end{tabular}

Cr: cockroach; Hu: Humulus scandens; Ra: ragweed pollen. * Jonckheere-Terpstra test: $p=0.229$.

spectrum due to differences in heredity, diet, and lifestyle. Zhang and Zhang [39] reviewed the sensitization pattern to inhalant allergens among AR patients in the mainland of China. They found that the prevalence and type of aeroallergens were different in diverse cities and regions. This study investigates the disease spectrum of allergic diseases accord- ing to the other areas and allergens by pooling data from 1367 patients with allergic diseases.

Although previous studies have shown the association of serum tIgE and sIgE levels with allergic diseases, few studies address this association in general. We have found that the mean tIgE in males was higher than that in females 


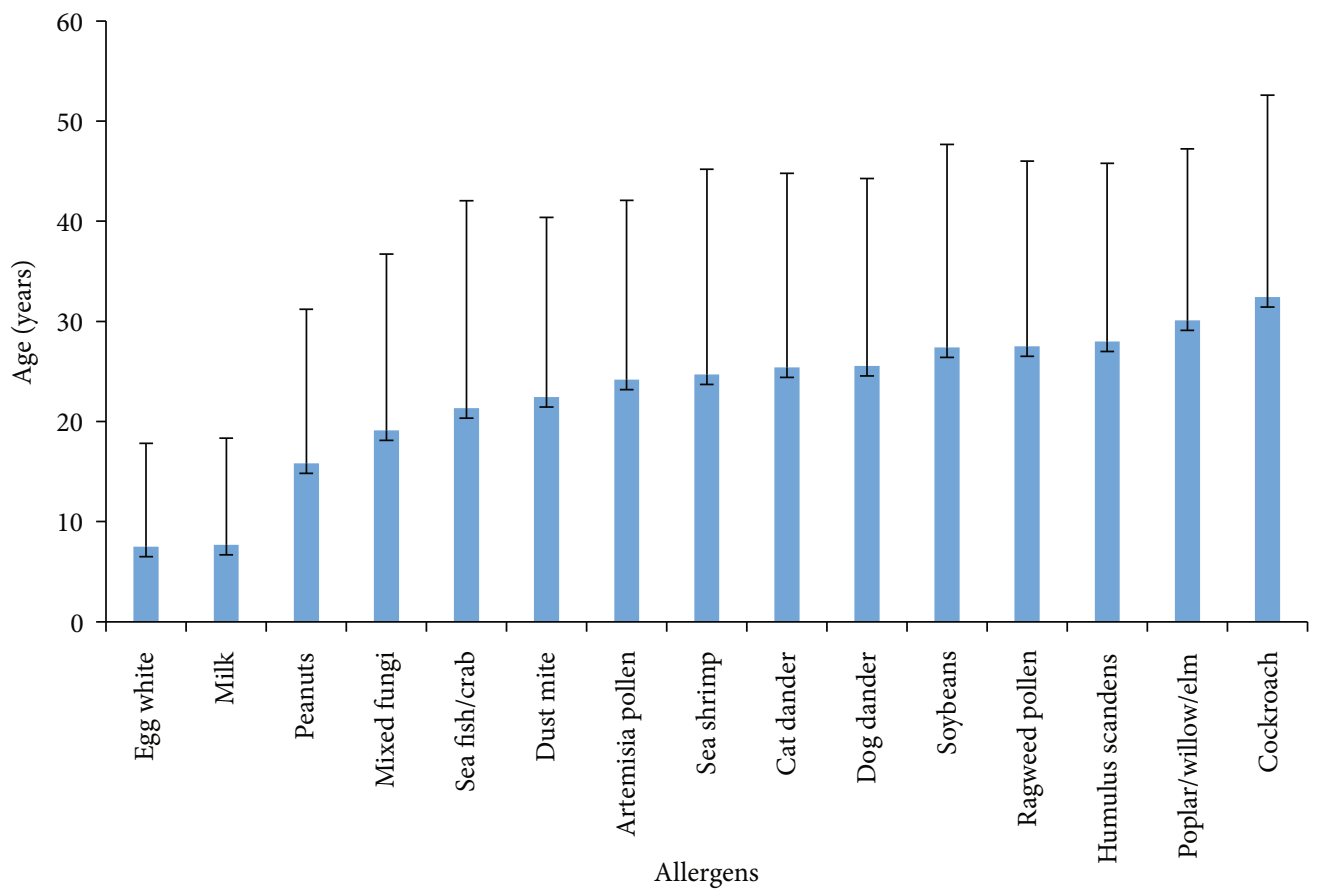

FIgURE 2: Average age of single allergen sensitization.

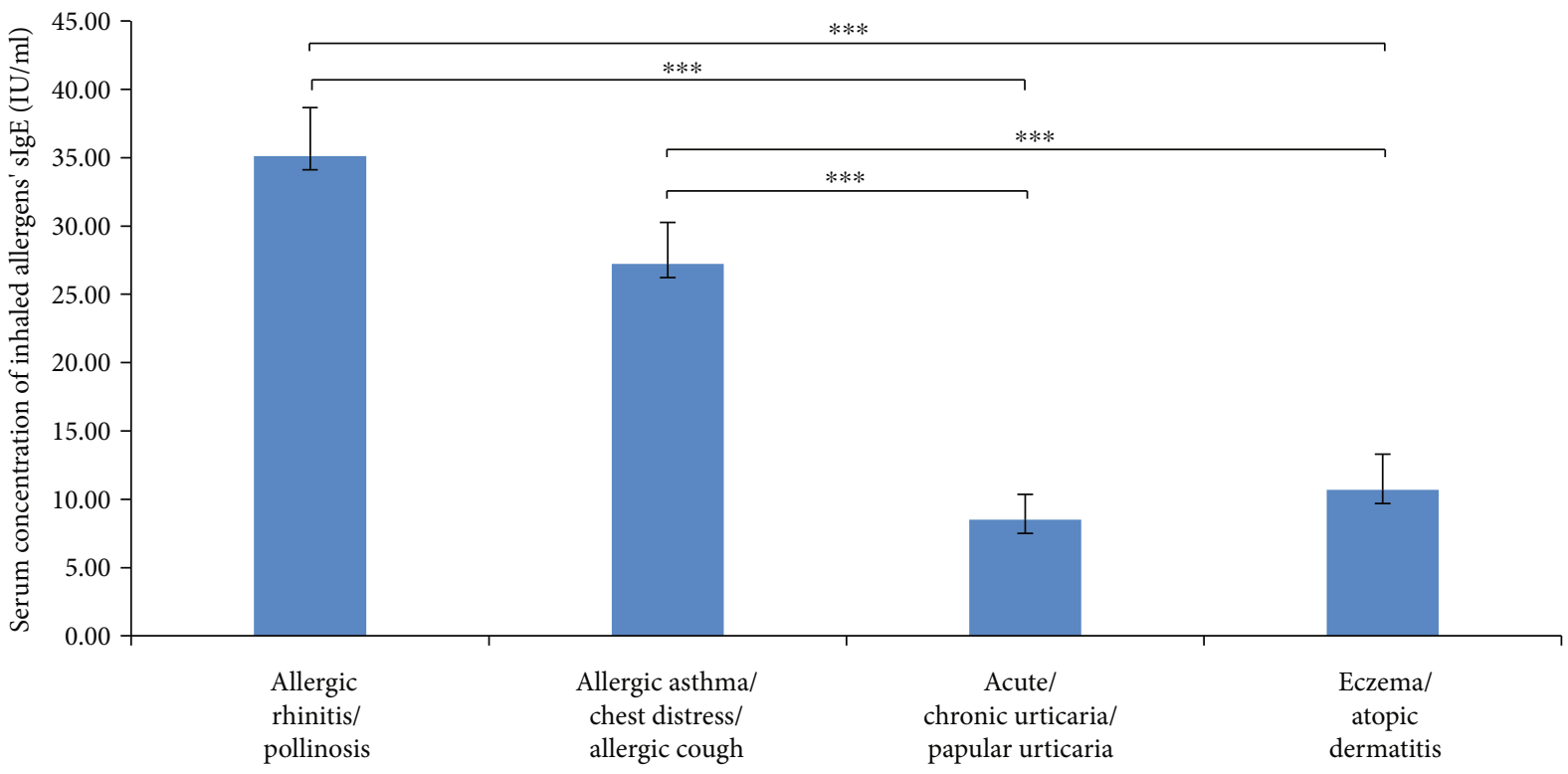

Figure 3: Mean value of inhaled allergen's sIgE in different diseases $\left({ }^{* * *} p<0.001\right)$.

$(p<0.05)$. We hypothesize that sex hormone may affect the level of serum IgE. In terms of age, the concentration of $\operatorname{tgE}$ is observed to increase from birth to adolescence and then decrease during adulthood, which has been validated in a previous study $[40,41]$. It has been suggested that the level of tIgE in Th2-driven respiratory inflammatory diseases is significantly higher than that in inflammatory skin diseases. Therefore, tIgE has developed as a Th2 biomarker, which is involved in regulating Th2 inflammatory response. The determination of serum tIgE in asthma patients can help determine the disease's severity, thus guiding the acute attack period's treatment and preventing remission [42].

The main allergens of respiratory allergy are dust mites, mixed fungi, Artemisia pollen, and Humulus scandens pollen. Dust mites are the main allergens in north China, consistent with other reports $[39,43]$. Limited by the types of allergens detected in the study, we do not have the data of the most common allergens in spring, such as Platanus 


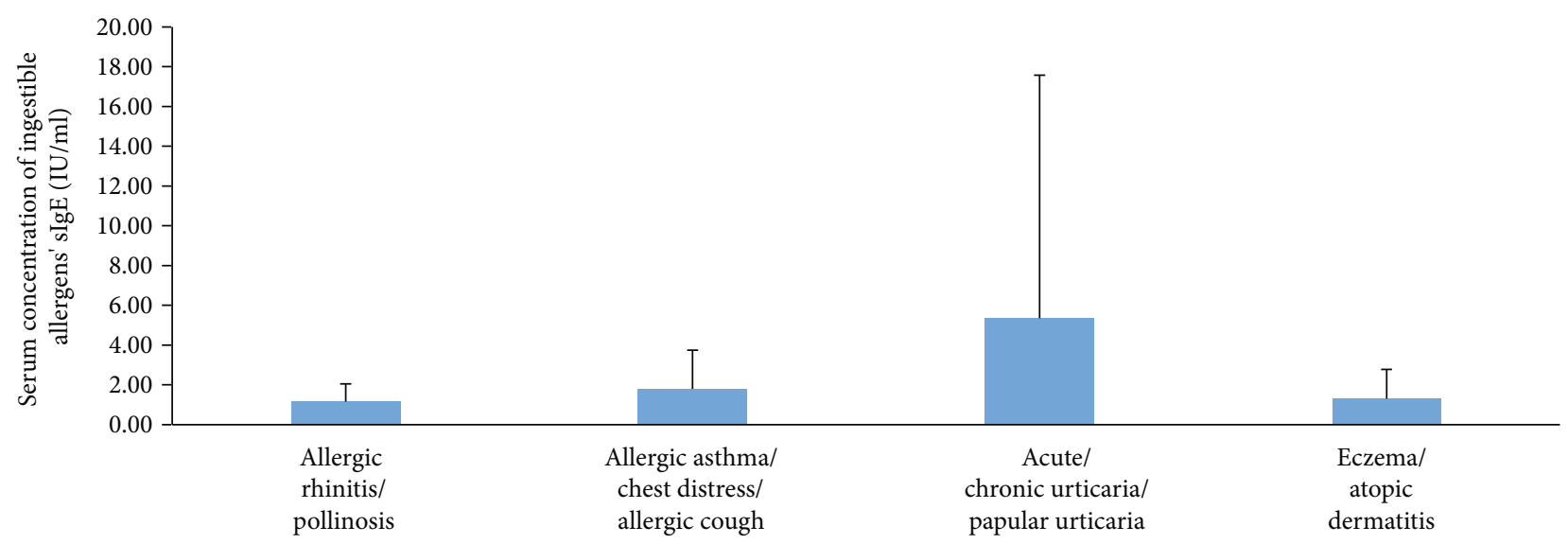

FIgURE 4: Mean value of ingestible allergen's sIgE in different diseases.

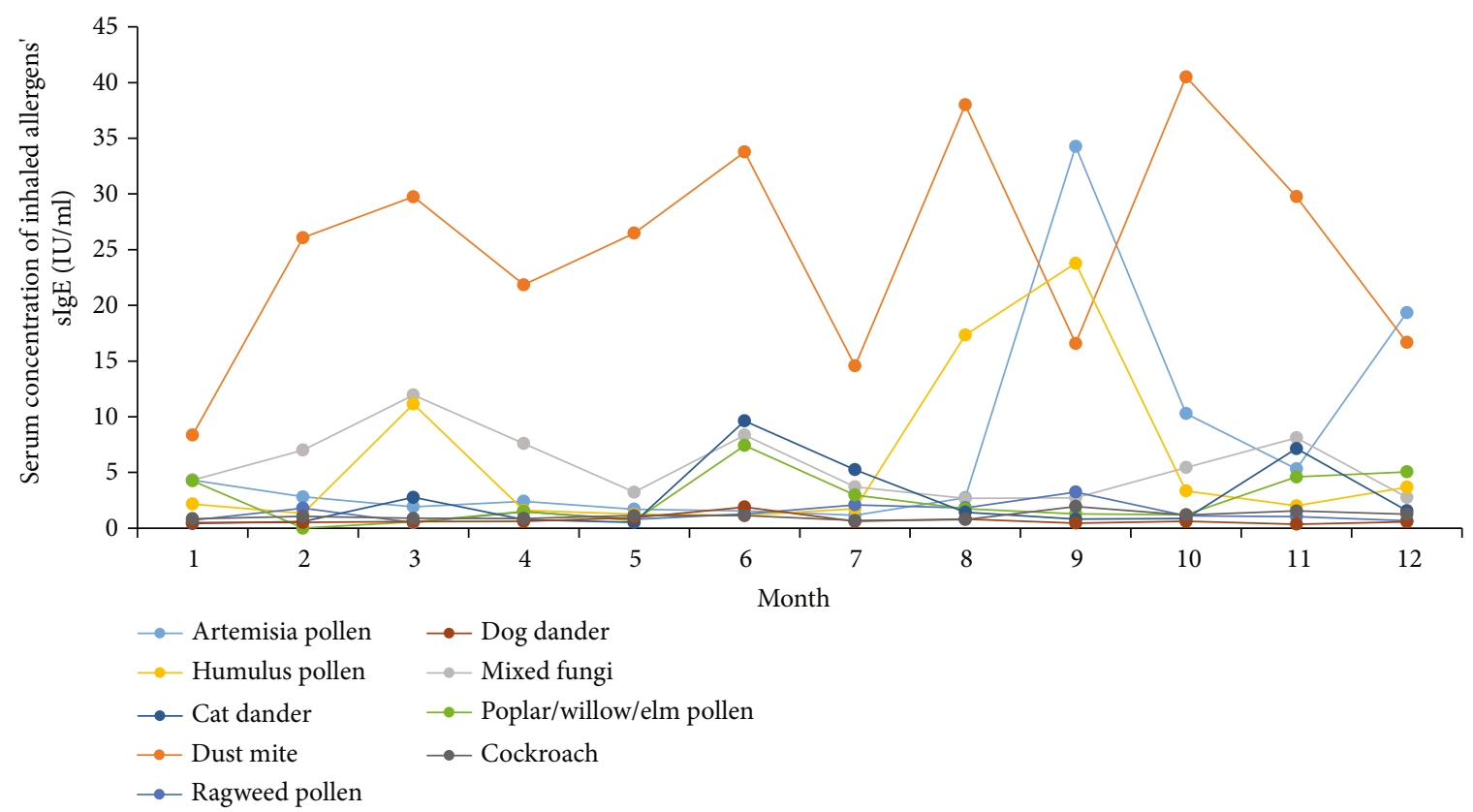

FIGURE 5: sIgE levels of 9 inhaled allergens in different months.

orientalis and ash tree pollen. Therefore, a large part of AR and pollinosis patients with negative sIgE and high $\operatorname{tgE}$ in spring are diagnosed by the skin prick test (SPT). At the same time, poplar/willows, which are native trees of north China, have visible white floccules but are rarely sensitized. For inflammatory skin diseases, urticaria accounts for $29.6 \%$ of the allergic population in this study, and $37 \%$ of them are detected as allergen-positive. Although the etiology of urticaria is complex, many relevant causes and/or triggers have been discovered, such as food in infants and children [44]. We have found that ingestion of many foods, including eggs, milk, and peanuts in sensitized infants/children, may cause acute urticaria more frequently than in adults. In adult urticaria, the IgE antibody in blood is more likely to be specific for inhalant allergens, such as dust mites and airborne pollen.

In this study, $87 \%$ of allergen-related eczema appeared after childhood. Food sensitization was only present in $21 \%$ of patients. $58 \%$ of the eczema population had more than one allergen. We also observed some IgE sensitization patterns, mainly including dust mites and molds, associated with persistent eczema/allergic dermatitis, especially in adults. The main food allergens for infantile eczema were still eggs and milk. Sensitization to sea fish/crab, sea shrimp, and peanuts among atopic children in Weifang was rarely observed. Although allergic patients will be refrained from eating spicy foods and seafood, we hypothesize that a nonIgE-mediated pathway may cause aggravation of dermatitis symptoms after seafood intake. Attributed to the gene differences with western populations, peanut and soybean nuts are not the leading causes of allergy in the Chinese population.

Among all immunoglobulin subclasses, IgE stands out concerning its low serum concentration and short half-life of 2 days. Seasonal allergen exposure can induce a rapid increase in allergen-specific IgE levels $[45,46]$. This study 


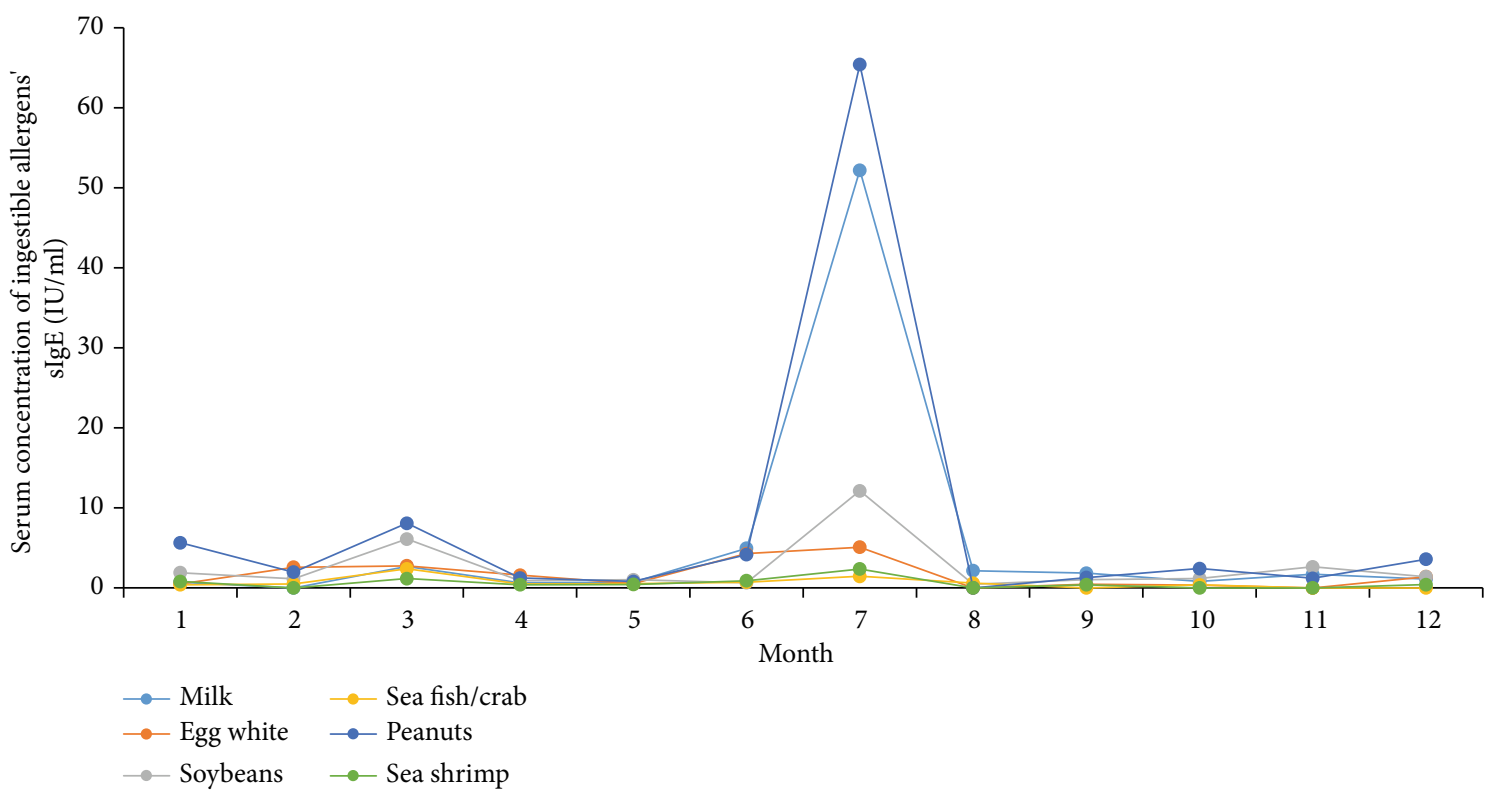

Figure 6: sIgE levels of 6 ingestible allergens in different months.

analyzed the time regularity of serum sIgE concentration of four main allergens, including dust mite, mixed fungi, Artemisia pollen, and Humulus pollen. There is a 1 month postponement between the serum sIgE concentration peak and the corresponding plant pollens' seasonal dissemination peak $[47,48]$. Although the environmental density data of dust mites and fungi are insufficient in Weifang, the role of meteorological factors in the survival and reproduction of fungi and dust mites is critical $[49,50]$. We speculate that the serum concentration of dust mites and fungi is closely related to seasonal reproduction. Therefore, it is necessary to detect airborne pollen and fungi as one of the routine meteorological forecast items and guide the allergic population to prevent and/or relocate to an allergen-free area for seasonal allergens.

Besides, sIgE levels of dust mites in inflammatory skin diseases (urticaria, eczema), classical type 2 inflammatory allergic diseases (AR and AS) are all statistically significant. The results have shown the allergic levels of dust mites in allergic skin patients were lower than those in respiratory allergic patients $(p<0.05)$. Similar findings have been found about fungi. There is a saying in China, namely, "one respiratory tract, one disease." We have found that AR patients tend to have higher sIgE antibodies than those with AS, especially in the two significant allergens of dust mites and Artemisia pollen. Recent cross-sectional studies have shown that the same individual can display different inflammation profiles in various respiratory tract sites [51]. There is an issue of whether each allergen family should be given a different cut-off. In addition to the most critical four allergens causing allergies, airborne pollen concentrations and food allergen antibodies are lower. However, their role in the allergy process is undeniable. This confusion was particularly evident after clinical verification of SPT.

Our study has some limitations. First, the patient's information is not further refined, including asthma, rhinitis, dermatitis symptom scores, and other indicators to assess the disease's severity. Change in IgE concentration with seasons has not been accurately counted. Second, the number of patients in each group is different. Almost half of the patients are diagnosed with urticaria and patients with $\mathrm{AD}$ account for only $3.2 \%$ of the sample. Third, the reagent kit does not contain the most common inhaled allergens of the Platanus orientalis in the north of China in spring and the ingestive allergens of wheat. Finally, because most of the patients are referred from Weifang, IgE's disease entity and measurements may not represent atopic patients' general population in Shandong province. More studies are warranted to elucidate the role of serological IgE detection of allergens according to different age, gender, and other factors in allergic diseases.

\section{Data Availability}

Data in this study is available from the corresponding author upon request.

\section{Conflicts of Interest}

All authors have no conflicts of interest to disclose.

\section{Acknowledgments}

We acknowledge funds from the Science and Technology Development Program of Weifang (2020YX013 and 2020YX084) and Shandong Science and Technology Development Program (2016WS0657).

\section{References}

[1] M. Andrea, B. Susanna, N. Francesca, M. Enrico, and V. Alessandra, "The emerging role of type 2 inflammation in asthma," Expert Review of Clinical Immunology, vol. 17, no. 1, pp. 63-71, 2021. 
[2] M. Kubo, "Innate and adaptive type 2 immunity in lung allergic inflammation,” Immunological Reviews, vol. 278, no. 1, pp. 162-172, 2017.

[3] L. M. Wheatley and A. Togias, "Clinical practice. Allergic rhinitis," The New England Journal of Medicine, vol. 372, no. 5, pp. 456-463, 2015.

[4] I. Agache, K. Sugita, H. Morita, M. Akdis, and C. A. Akdis, "The complex type 2 endotype in allergy and asthma: from laboratory to bedside," Current Allergy and Asthma Reports, vol. 15, no. 6, p. 29, 2015.

[5] A. V. Kudryavtseva, K. A. Neskorodova, and P. Staubach, "Urticaria in children and adolescents: an updated review of the pathogenesis and management," Pediatric Allergy and Immunology, vol. 30, no. 1, pp. 17-24, 2019.

[6] J. Zhao, J. Bai, K. Shen et al., "Self-reported prevalence of childhood allergic diseases in three cities of China: a multicenter study," BMC Public Health, vol. 10, no. 1, 2010.

[7] N. Ravnborg, D. Ambikaibalan, G. Agnihotri et al., "Prevalence of asthma in patients with atopic dermatitis: a systematic review and meta-analysis," Journal of the American Academy of Dermatology, vol. 84, no. 2, pp. 471-478, 2021.

[8] F. Li, Y. Zhou, S. Li et al., "Prevalence and risk factors of childhood allergic diseases in eight metropolitan cities in China: a multicenter study," BMC Public Health, vol. 11, no. 1, 2011.

[9] W. Feleszko, M. Ruszczy ski, J. Jaworska, A. Strzelak, B. M. Zalewski, and M. Kulus, "Environmental tobacco smoke exposure and risk of allergic sensitisation in children: a systematic review and meta-analysis," Archives of Disease in Childhood, vol. 99, no. 11, pp. 985-992, 2014.

[10] J. S. Kim, F. Ouyang, J. A. Pongracic et al., "Dissociation between the prevalence of atopy and allergic disease in rural china among children and adults," Journal of Allergy and Clinical Immunology, vol. 122, no. 5, pp. 929-935.e4, 2008.

[11] B. Q. Sun, D. H. Chen, P. Y. Zheng et al., "Allergy-related evidences in relation to serum ige: data from the China state key laboratory of respiratory disease, 2008-2013," Biomedical and Environmental Sciences, vol. 27, no. 7, pp. 495-505, 2014.

[12] S. Lee, "Ige-mediated food allergies in children: prevalence, triggers, and management," Korean Journal of Pediatrics, vol. 60, no. 4, pp. 99-105, 2017.

[13] C. H. Katelaris, B. W. Lee, P. C. Potter et al., "Prevalence and diversity of allergic rhinitis in regions of the world beyond europe and north america," Clinical and Experimental Allergy, vol. 42, no. 2, pp. 186-207, 2012.

[14] H. T. Wang, C. M. Warren, R. S. Gupta, and C. M. Davis, "Prevalence and characteristics of shellfish allergy in the pediatric population of the united states," The Journal of Allergy and Clinical Immunology: In Practice, vol. 8, no. 4, pp. 13591370.e2, 2020.

[15] J. Stern, J. Pier, and A. A. Litonjua, "Asthma epidemiology and risk factors," Seminars in Immunopathology, vol. 42, no. 1, pp. 5-15, 2020.

[16] M. J. Ridd, A. J. L. King, E. le Roux, A. Waldecker, and A. L. Huntley, "Systematic review of self-management interventions for people with eczema," The British Journal of Dermatology, vol. 177, no. 3, pp. 719-734, 2017.

[17] A. Muraro, G. Roberts, M. Worm et al., "Anaphylaxis: guidelines from the european academy of allergy and clinical immunology," Allergy, vol. 69, no. 8, pp. 1026-1045, 2014.

[18] F. E. Simons, M. Ebisawa, M. Sanchez-Borges et al., “2015 update of the evidence base: world allergy organization ana- phylaxis guidelines," World Allergy Organization Journal, vol. 8, no. 1, p. 32, 2015.

[19] M. A. Tejedor Alonso, M. Moro Moro, and M. V. Múgica García, "Epidemiology of anaphylaxis," Clinical and Experimental Allergy, vol. 45, no. 6, pp. 1027-1039, 2015.

[20] W. Luo, H. Hu, W. Tang et al., “Allergen sensitization pattern of allergic adults and children in southern China: a survey based on real life data," Allergy, Asthma and Clinical Immunology, vol. 15, no. 1, 2019.

[21] J. Wang, Z. Zhao, Y. Zhang et al., "Asthma, allergic rhinitis and eczema among parents of preschool children in relation to climate, and dampness and mold in dwellings in China," Environment International, vol. 130, p. 104910, 2019.

[22] X. D. Wang, M. Zheng, H. F. Lou et al., "An increased prevalence of self-reported allergic rhinitis in major chinese cities from 2005 to 2011," Allergy, vol. 71, no. 8, pp. 1170-1180, 2016.

[23] H. Mosbech, N. H. Nielsen, A. Dirksen, J. Launbjerg, I. Biering, and M. Søborg, "Comparison between specific ige measured by rast, two chemiluminescent assays and skin prick test," Allergologia et Immunopathologia, vol. 20, no. 6, pp. 220-224, 1992.

[24] L. Cheng, J. Chen, Q. Fu et al., "Chinese society of allergy guidelines for diagnosis and treatment of allergic rhinitis," Allergy, Asthma \& Immunology Research, vol. 10, no. 4, pp. 300-353, 2018.

[25] N. G. Papadopoulos, H. Arakawa, K. H. Carlsen et al., "International consensus on (icon) pediatric asthma," Allergy, vol. 67, no. 8, pp. 976-997, 2012.

[26] T. Bieber, "Atopic dermatitis," The New England Journal of Medicine, vol. 358, no. 14, pp. 1483-1494, 2008.

[27] A. Wollenberg, S. Barbarot, T. Bieber et al., "Consensus-based european guidelines for treatment of atopic eczema (atopic dermatitis) in adults and children: part ii," Journal of the European Academy of Dermatology and Venereology, vol. 32, no. 6, pp. 850-878, 2018.

[28] R. A. Wood, D. A. Khan, D. M. Lang et al., "American Academy of Allergy, Asthma and Immunology response to the EAACI/GA2LEN/EDF/WAO guideline for the definition, classification, diagnosis, and management of urticaria 2017 revision," Allergy, vol. 74, no. 2, pp. 411-413, 2019.

[29] J. Schmitt, S. Langan, S. Deckert et al., “Assessment of clinical signs of atopic dermatitis: a systematic review and recommendation," The Journal of Allergy and Clinical Immunology, vol. 132, no. 6, pp. 1337-1347, 2013.

[30] F. E. Simons and H. A. Sampson, "Anaphylaxis epidemic: fact or fiction?," The Journal of Allergy and Clinical Immunology, vol. 122, no. 6, pp. 1166-1168, 2008.

[31] J. Ščevková, J. Dušička, M. Hrubiško, and K. Mičieta, "Influence of airborne pollen counts and length of pollen season of selected allergenic plants on the concentration of sige antibodies on the population of Bratislava, Slovakia," Annals of Agricultural and Environmental Medicine, vol. 22, no. 3, pp. 451-455, 2015.

[32] S. C. Dharmage, A. J. Lowe, M. C. Matheson, J. A. Burgess, K. J. Allen, and M. J. Abramson, "Atopic dermatitis and the atopic march revisited," Allergy, vol. 69, no. 1, pp. 17-27, 2014.

[33] S. Illi, E. von Mutius, S. Lau et al., "The natural course of atopic dermatitis from birth to age 7 years and the association with asthma," The Journal of Allergy and Clinical Immunology, vol. 113, no. 5, pp. 925-931, 2004.

[34] A. S. Paller, J. M. Spergel, P. Mina-Osorio, and A. D. Irvine, "The atopic march and atopic multimorbidity: many 
trajectories, many pathways," The Journal of Allergy and Clinical Immunology, vol. 143, no. 1, pp. 46-55, 2019.

[35] M. Saunes, T. Øien, C. K. Dotterud et al., "Early eczema and the risk of childhood asthma: a prospective, populationbased study," BMC Pediatrics, vol. 12, no. 1, 2012.

[36] K. A. Engebretsen and J. P. Thyssen, Skin barrier function and allergens, vol. 49 of Current Problems in Dermatology, Basel, Karger, 2016.

[37] M. Shaker, "New insights into the allergic march," Current Opinion in Pediatrics, vol. 26, no. 4, pp. 516-520, 2014.

[38] D. C. Belgrave, R. Granell, A. Simpson et al., "Developmental profiles of eczema, wheeze, and rhinitis: two populationbased birth cohort studies," PLoS Medicine, vol. 11, no. 10, p. e1001748, 2014.

[39] Y. Zhang and L. Zhang, "Prevalence of allergic rhinitis in China," Allergy, Asthma \& Immunology Research, vol. 6, no. 2, pp. 105-113, 2014.

[40] R. A. Barbee, M. Halonen, M. Lebowitz, and B. Burrows, "Distribution of ige in a community population sample: correlations with age, sex, and allergen skin test reactivity," The Journal of Allergy and Clinical Immunology, vol. 68, no. 2, pp. 106-111, 1981.

[41] J. W. Gerrard, S. Horne, P. Vickers et al., "Serum ige levels in parents and children," The Journal of Pediatrics, vol. 85, no. 5, pp. 660-663, 1974.

[42] K. Kovač, S. Dodig, D. Tješić-Drinković, and M. Raos, “Correlation between Asthma Severity and Serum IgE in Asthmatic Children Sensitized to _Dermatophagoides pteronyssinus_," Archives of Medical Research, vol. 38, no. 1, pp. 99-105, 2007.

[43] J. Li, B. Sun, Y. Huang et al., "A multicentre study assessing the prevalence of sensitizations in patients with asthma and/or rhinitis in China," Allergy, vol. 64, no. 7, pp. 1083-1092, 2009.

[44] O. Jirapongsananuruk, S. Pongpreuksa, P. Sangacharoenkit, N. Visitsunthorn, and P. Vichyanond, "Identification of the etiologies of chronic urticaria in children: a prospective study of 94 patients," Pediatric Allergy and Immunology, vol. 21, no. 3, pp. 508-514, 2010.

[45] J. Eckl-Dorna, S. Villazala-Merino, N. J. Campion et al., "Tracing ige-producing cells in allergic patients," Cells, vol. 8, no. 9, p. 994, 2019.

[46] V. Niederberger, J. Ring, J. Rakoski et al., "Antigens drive memory ige responses in human allergy via the nasal mucosa," International Archives of Allergy and Immunology, vol. 142, no. 2, pp. 133-144, 2007.

[47] G. J. Gleich, G. L. Jacob, J. W. Yunginger, and L. L. Henderson, "Measurement of the absolute levels of IgE antibodies in patients with ragweed hay fever: effect of immunotherapy on seasonal changes and relationship to IgG antibodies," The Journal of Allergy and Clinical Immunology, vol. 60, no. 3, pp. 188-198, 1977.

[48] Y. Ouyang, Z. Yin, Y. Li, E. Fan, and L. Zhang, "Associations among air pollutants, grass pollens, and daily number of grass pollen allergen-positive patients: a longitudinal study from 2012 to 2016," International Forum of Allergy \& Rhinology, vol. 9, no. 11, pp. 1297-1303, 2019.

[49] A. A. Abdel Hameed, M. I. Khoder, Y. H. Ibrahim, Y. Saeed, M. E. Osman, and S. Ghanem, "Study on some factors affecting survivability of airborne fungi," Science of The Total Environment, vol. 414, pp. 696-700, 2012.
[50] J. D. Miller, "The role of dust mites in allergy," Clinical Reviews in Allergy and Immunology, vol. 57, no. 3, pp. 312-329, 2019.

[51] L. Giovannini-Chami, A. Paquet, C. Sanfiorenzo et al., "The "one airway, one disease" concept in light of th2 inflammation," European Respiratory Journal, vol. 52, no. 4, p. 1800437, 2018. 